\title{
Fungi Associated with Maize and Sorghum Grains and their Potential for Amylase and Aflatoxins Production
}

\author{
M.A. Abdel- Sater ${ }^{1}$, S.I.I.Abdel- Hafez ${ }^{1}$, Nemmat, A. \\ Hussein $^{1 *}$ and Eshraq, A. AL-Amery ${ }^{1,2}$ \\ ${ }^{1}$ Botany and Microbiology Department, Faculty of Science, \\ Assiut University, Assiut and ${ }^{2}$ Microbiology Department, \\ Faculty of Applied Science, Taiz University, Yemen.
}

\begin{abstract}
【SING direct-plating technique, thirty-nine species belonging to 16 fungal genera were isolated from maize and sorghum grain samples (20 samples each) on Czapek's agar (Cz) and Czapek's supplemented with $40 \%$ sucrose agar $(\mathrm{Cz} 40 \mathrm{~S})$ media at $28^{\circ} \mathrm{C}$. Widest spectrum of genera and species were recorded on sorghum (16 genera and 35 species) compared with maize grains (8 and 19). The highest total count (139 CFUs/ 100 grains) and the number of genera (14) and species (27) were identified from sorghum grains on $\mathrm{Cz}$ medium. The most common fungi on the grains tested were Aspergillus flavus, A. niger, Eurotium amstelodami, E. rubrum, E. repens, Fusarium verticillioides and Rhizopus stolonifer. Among 129 isolates screened for their abilities to produce amylase enzyme, 102 isolates could produce this enzyme, of which $A$. terreus exhibited the highest production (EI=1.73). HPLC analysis revealed that out of 6 strains of A. flavus tested, strain No. AUMC 11311 showed the highest production of aflatoxin $B_{1}$ and $B_{2}$ while the highest value of aflatoxin $\mathrm{G}_{2}$ was produced by strain No. AUMC 11317 . It could be concluded that fungi growing on grains and have the abilities of producing enzymes and/or aflatoxins might cause deterioration and spoilage to these grains.
\end{abstract}

Keywords: Fungi, Deterioration, Sorghum, Maize, Aflatoxins, Amylase.

Maize is the third most important food crop in the world surpassed only by wheat and rice. Yemen production of maize is estimated 221,078 tons according to the Statistical Agricultural Center in 2013. Alternaria alternata, Aspergillus candidus, A. flavus, A. fumigatus, A. niger, A. ochraceus, A. parasiticus, Bipolaris maydis, Chaetomium globosum, Cladosporium cladosporioides, Cochlibolus lunata, Drechslera halodes, Epicoccum, Fusarium culmorum, F. graminearum, $F$. oxysporum, $F$. proliferatum, $F$. semitectum, Penicillium citrinum, P. funiculosum, P. oxalicum, Phoma herbarum, Rhizopus oryzae, $R$. stolonifer, Rhizoctonia solani and Trichoderma harzianum were isolated from maize grains in Egypt (Ismail et al., 2016), Pakistan, (Niaz and Dawar 2009), Ethiopia (Ofgea and Gure, 2015) and Colombo (Abe et al., 2015).

Sorghum is located the fifth among cereal crops with 60 million tons

*Corresponding author email: nemmgoda@yahoo.com 
annually produced in the world. It is the staple food grain for over 750 million people in Africa, Asia, and Latin America (Wilson et al., 1995). Several studies on fungi associated with sorghum grains, were done and noticed that Alternaria, Arthrinium, Aspergillus Botrytis, Cercosporella, Cladosporium, Colletotrichum,, Curvularia, Phoma, Rhizopus, Fusarium, Humicola and Trichoderma were isolated (Abdel-Hafez et al., 2014, Mohammed et al., 2015 and Machio 2016).

One of the main ways of fungal attack of the grain is that production of cell wall- degrading enzymes. Several filamentous fungi have proven to be an important source of hydrolytic enzymes (Ogbonna et al., 2015). Aspergillus flavus, A. fumigatus, A. niger, A. ochraceus, A. oryzae and A. terreus, Emericella nidulans, Mucor racemosus, Mycosphaerella tassiana, Penicillium chrysogenum, Rhizopus oligosporus, R. stolonifer and several others are used as sources of fungal $\alpha$ - amylases (Irfan et al., 2012 and Singh et al., 2014). In addition, amylases have immense applications on various fields in world market because of their wide applications in industries including food, brewing, distilling industry, textile, paper, pharmaceutical and bioconversion of solid wastes (Lall et al. 2015).

The other way of fungi to deteriorate the grains is by forming mycotoxins. Aflatoxins are natural secondary metabolites produced mainly by Aspergillus flavus group (section Flavi) that contaminant agricultural commodities in the field particularly in critical temperature and humidity conditions before or during harvest or in storage (Rustom, 1997 and Sweeney and Dobson 1998). Aflatoxins are classified to $\mathrm{B}_{1}\left(\mathrm{AFB}_{1}\right)$ and $\mathrm{B}_{2}\left(\mathrm{AFB}_{2}\right)$, produced by $A$. flavus and $\mathrm{G}_{1}\left(\mathrm{AFG}_{1}\right)$ and $\mathrm{G}_{2}$ $\left(\mathrm{AFG}_{2}\right)$, produced by $A$. flavus as well as A. parasiticus. The WHO-International Agency for Research on Cancer (IARC) has classified all types of aflatoxins as carcinogenic agents to humans (IARC, 2002). Aflatoxins are largely associated with commodities produced in the tropics and subtropics crops, such as cotton, peanuts, pistachios, sorghum and maize (Yin et al., 2008 and Kange et al., 2015).

The objective of the current study was to assess the fungal diversity associated with maize and sorghum grains collected from Taiz Governorate, Yemen. Amylase production by isolated fungi and aflatoxigenic potential of some Aspergillus flavus strains were also evaluated.

\section{Material and Methods}

\section{Collection of samples}

Forty samples of maize (20 samples) and sorghum grains (20) were collected from different markets and local stores, in Taiz Governorate, Yemen (Table 1). The samples were put in clean polyethylene plastic bags, brought to the Mycological Laboratory and kept at $5^{\circ} \mathrm{C}$ till fungal analysis. 
The moisture contents of grain samples were estimated using the method described by Abdel-Hafez et al. (2014) and expressed as percentage of dry weight.

\section{Germinability test}

The viability of grains was assessed through their ability for germination. Filter papers were placed inside the Petri-dishes, moisten the paper until there was a tiny bit of standing water. Ten grains were put above the wet paper in each plate (Gummert, 2011). Put the plates in a warm place $\left(30^{\circ} \mathrm{C}\right)$. Check the germination of grains every day, count germinated grain seedlings and the percentage of germination was calculated as following:

$$
\% \text { germination }=\frac{\text { No. of germination seeds }}{\text { No. o } \square \text { total seeds planted }} \times 100
$$

\section{Isolation and identification of fungi}

The direct-plating technique was used to determine grain-borne fungi of maize and sorghum. Czapek's (Cz) and Czapek's supplemented with $40 \%$ sucrose (Cz40S) agar media incubated at $28^{\circ} \mathrm{C}$ were used for isolation of fungi. Five grains of each sample were placed directly on the surface of each agar plate (Pitt $e t$ al., 1992). Four-replicate plates were used for each medium and the plates were incubated at $28^{\circ} \mathrm{C}$ for 7 days. The developing fungi were counted0, isolated, identified and calculated as colony forming units (CFUs) per 20 grains for each sample. Isolated fungi were identified mainly on the basis of their macro- and microscopic features following the keys of Raper and Fennell (1965), Booth (1971), Ellis (1971), Pitt (1979), Moubasher (1993), Leslie and Summerell (2006), Domsch et al. (2007), Pitt and Hocking (2009) and Ismail et al. (2015).

\section{Screening for $\alpha$ - amylase production}

One hundred and twenty-nine isolates collected from maize (41 isolates) and sorghum grains (88) were assayed for their abilities to produce $\alpha$ - amylase on modified Czapek's agar medium with the following composition (g/l): starch, 30; $\mathrm{NaNO}_{3}, 3 ; \mathrm{KH}_{2} \mathrm{PO}_{4}, 1.0 ; \mathrm{MgSO}_{4} .7 \mathrm{H}_{2} \mathrm{O}, 0.5 ; \mathrm{KCL}, 0.5 ; \mathrm{FeSO}_{4}, 0.01 ;$ agar, 15 and distilled water, $1000 \mathrm{ml}$ (Bridge, 1985). The inoculated agar plates were incubated at $28^{\circ} \mathrm{C}$ for 5-7 days, then flooded with iodine solution. A clear zone around fungal growth indicates the production of $\alpha$-amylase (Cowan, 1974). Enzyme index (EI) was calculated according to Ho and Foster (1972) as follows:

\footnotetext{
Diameter of the outer limited of the clear $\mathrm{Z} \square$ ne

Enzyme index $(\mathrm{EI})=$ Diameter of the fungal colony

Screening for aflatoxins
} 
Nineteen isolates of Aspergillus flavus, A. flavus var. columnaris and A. tamarii, collected during the current work, were screened for their aflatoxin potential using coconut agar medium (CAM). $100 \mathrm{~g}$ of shredded coconut were homogenized for $5 \mathrm{~min}$ with $300 \mathrm{ml}$ hot distilled water, then filtered and the

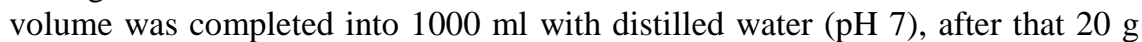
agar were added. After sterilization, $15-20 \mathrm{ml}$ was poured into sterile Petridishes. Fungal isolates were inoculated at the center of CAM agar plates and incubated at $28^{\circ} \mathrm{C}$ in the dark for 7 days. Cultures were observed for fluorescence under UV light $(365 \mathrm{~nm})$. The positive results were detected as blue fluorescence and an uninoculated plate was observed as a reference (Davis et al., 1987).

\section{Extraction of aflatoxins from fungal isolates}

To determine production of aflatoxin, six isolates of fungi were inoculated on potato dextrose broth. After 10 days incubation period, the content of each flask was homogenized with $100 \mathrm{ml}$ chloroform, washed with equal volume of distilled water, dried over anhydrous sodium sulphate, filtered then concentrated under vaccum to near dryness, and diluted with $1 \mathrm{ml}$ chloroform (Pons et al., 1972).

\section{Assessment of aflatoxins by HPLC}

For determination of aflatoxin from six fungal isolates, silica-based HPLC columns bonded with $\mathrm{C} 8$ or $\mathrm{C} 18$ groups are used with mobile phases consisting of binary or ternary mixtures of polar solvents. Commonly used solvent (deionized water, methanol and acetonitrile). In the reversed phase mode, the elution order of the common aflatoxins is $\mathrm{B}_{1}, \mathrm{~B}_{2}$ and $\mathrm{G}_{2}$ (Sekar et al. 2008).

\section{Results}

\section{Moisture contents of the grains}

Results in Table 1 showed that, moisture content of sorghum grains (10.6$20.2 \%)$ were much higher than that of maize grains $(8.9-11.2 \%)$. The highest value of MC\% for sorghum grains was recorded in sample number 37, while the lowest value was detected in sample No. 22. On the other hand, maize sample No. 11 showed the highest moisture content, while, Nos. 4 and 20 recorded the least $\mathrm{MC} \%$ values.

\section{Germinability of the grains}

The percentage of germination for 20 grain samples ranging between, 40$100 \%$ of maize and $30-90 \%$ of sorghum grains. Maize grain Nos. 8, 10, 18, 20 gave $100 \%$ germination. While, the highest germinability of sorghum grains (90\%) were detected in samples Nos. 28 and 35. On the other hand, low values of germinability were recorded in sorghum grain sample No. 30 and in maize grain sample No. 9 (Table 1). 
TABLE 1. Localities, percentage moisture content (MC\%) and percentage germinability $(\mathrm{G} \%)$ of maize and sorghum grain samples.

\begin{tabular}{|l|c|c|c|c|c|c|}
\multirow{2}{*}{ Locality } & \multicolumn{5}{c|}{ Maize } & \multicolumn{3}{c|}{ Sorghum } \\
\cline { 2 - 7 } & No. & MC\% & G\% & No. & MC\% & G\% \\
\hline Alshenany & 1 & 10.1 & 70 & 21 & 14 & 50 \\
\hline Alshenany & 2 & 10.8 & 60 & 22 & 10.6 & 70 \\
\hline Alshenany & 3 & 9.1 & 60 & 23 & 11.6 & 60 \\
\hline Alshenany & 4 & 8.9 & 80 & 24 & 14.2 & 80 \\
\hline Alshenany & 5 & 9.7 & 90 & 25 & 11 & 80 \\
\hline Alsamsara & 6 & 9.3 & 70 & 26 & 15.2 & 70 \\
Alsamsara & 7 & 9.2 & 90 & 27 & 12.5 & 80 \\
\hline Alsamsara & 8 & 9.6 & 100 & 28 & 13.2 & 90 \\
Alsamsara & 9 & 10.1 & 40 & 29 & 13.2 & 50 \\
\hline Alsamsara & 10 & 10.5 & 100 & 30 & 14.2 & 30 \\
\hline Alsamsara & 11 & 11.2 & 70 & 31 & 14.8 & 80 \\
\hline Bearbasha & 12 & 10.5 & 60 & 32 & 12.5 & 60 \\
\hline Bearbasha & 13 & 11 & 70 & 33 & 12.4 & 70 \\
\hline Bearbasha & 14 & 9.6 & 80 & 34 & 11.9 & 80 \\
\hline ALaamor village & 15 & 10.8 & 80 & 35 & 12.7 & 90 \\
\hline ALaamor village & 16 & 9.3 & 70 & 36 & 13.4 & 80 \\
\hline ALaamor village & 17 & 9.7 & 90 & 37 & 20.2 & 70 \\
\hline 26 September Street & 18 & 10.5 & 100 & 38 & 12.7 & 50 \\
\hline 26 September Street & 19 & 10.1 & 50 & 39 & 14.3 & 80 \\
\hline 26 September Street & 20 & 8.9 & 100 & 40 & 14.6 & 70 \\
\hline
\end{tabular}

Fungi recovered in the present investigation

All sorghum grain samples were contaminated with fungi, while 17 maize samples (out of 20) were contaminated by fungi. Thirty-nine species belonging to 16 fungal genera were isolated from maize and sorghum grain samples on Czapek's agar (Cz) and Czapek's supplemented with 40\% sucrose agar (Cz40S) media. The total number of genera (16) and species (35) recorded on sorghum were highly significant than those obtained on maize (8 genera and 19 species) (Tables 1, 2, 3, 4 \& Fig. 1,2).

TABLE 2. Mean \pm standard error of total fungal counts and number of species isolated from maize and sorghum grains on $\mathrm{Cz}$ and $\mathrm{Cz} 40 \mathrm{~S}$ agar media.

\begin{tabular}{|r|c|c|c|c|c|c|}
\hline $\begin{array}{c}\text { Source \& } \\
\text { Media }\end{array}$ & Aspergillus & Eurotium & Fusarium & Penicillium & Total count & No of species \\
\hline Maize Cz & $102.75 \pm 13.4^{\mathrm{b}}$ & $2 \pm 0.4^{\mathrm{a}}$ & $19.5 \pm 2.8^{\mathrm{c}}$ & $10.5 \pm 3.1^{\mathrm{b}}$ & $121 \pm 2.3^{\mathrm{a}}$ & $7.25 \pm 0.6^{\mathrm{a}}$ \\
\cline { 2 - 7 } $\mathbf{C z 4 0}$ & $69.75 \pm 3.1^{\mathrm{a}}$ & $45.25 \pm 3.1^{\mathrm{b}}$ & $00 \pm 00^{\mathrm{a}}$ & $10 \pm 2.3^{\mathrm{b}}$ & $125.75 \pm 7.2^{\mathrm{ab}}$ & $7.75 \pm 0.3^{\mathrm{a}}$ \\
\hline Sorghum Cz & $89.25 \pm 2.9^{\mathrm{ab}}$ & $7.25 \pm 3.0^{\mathrm{a}}$ & $5.75 \pm 1.3^{\mathrm{b}}$ & $1.5 \pm 0.9^{\mathrm{a}}$ & $138.25 \pm 2.3^{\mathrm{b}}$ & $10.25 \pm 0.5^{\mathrm{b}}$ \\
\hline $\mathbf{C z 4 0}$ & $72 \pm 2.0^{\mathrm{a}}$ & $58.25 \pm 1.5^{\mathrm{c}}$ & $0.25 \pm 0.3^{\mathrm{a}}$ & $0.75 \pm 0.8^{\mathrm{a}}$ & $138 \pm 1.2^{\mathrm{b}}$ & $10.25 \pm 0.5^{\mathrm{b}}$ \\
\hline F & $\mathbf{4 . 8}^{*}$ & $\mathbf{1 4 5 . 9 * *}$ & $\mathbf{3 4 . 4}^{* *}$ & $\mathbf{7 . 0 2}^{* *}$ & $\mathbf{4 . 8}^{*}$ & $\mathbf{1 1 . 2}^{* *}$ \\
\hline
\end{tabular}


Aspergillus, Eurotium, Fusarium and sterile mycelia possessed more propagules on sorghum than on maize, while, Penicillium had highly significant propagules on maize than on sorghum. The total viable counts of fungi in sorghum on $\mathrm{Cz}$ (139 CFUs/ 100 grains) and on Cz40S (138) were significant than those in maize on Cz40S (126 CFUs/ 100 grains) and on $\mathrm{Cz}$ (121) (Tables $2,3)$. The highest total fungal count was recorded in sorghum grains on $\mathrm{Cz}$ agar medium (139 CFUs/ 100 grains), followed by those isolated on Cz40S (138 CFUs per 100 sorghum grains) (Fig. 1,2).

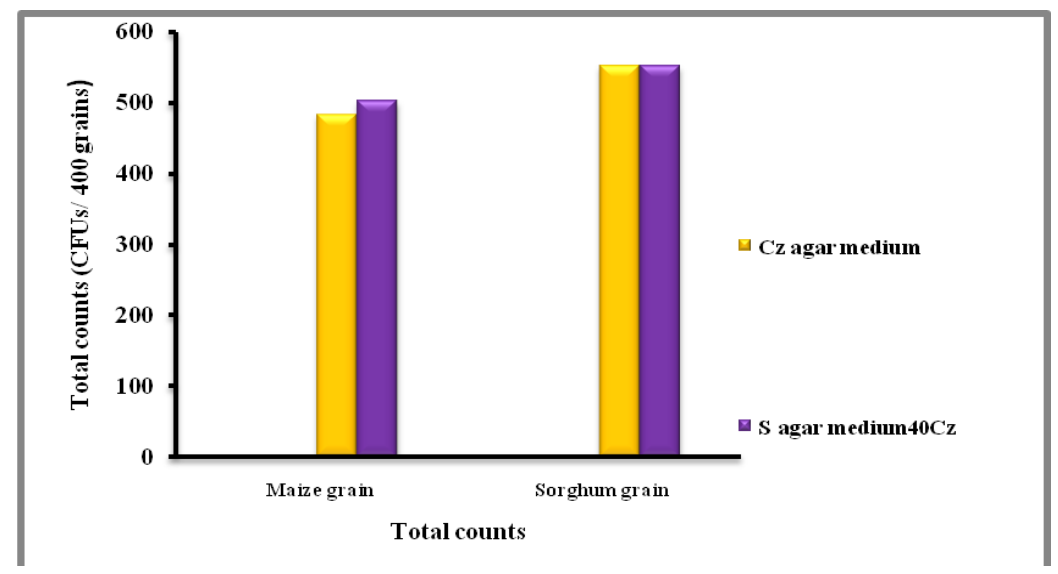

Fig. 1. Diagram illustrating the total counts of fungi isolated from maize and sorghum grains (per 100 grains) on $\mathrm{Cz}$ and $\mathrm{Cz} 40 \mathrm{~S}$ agar media.

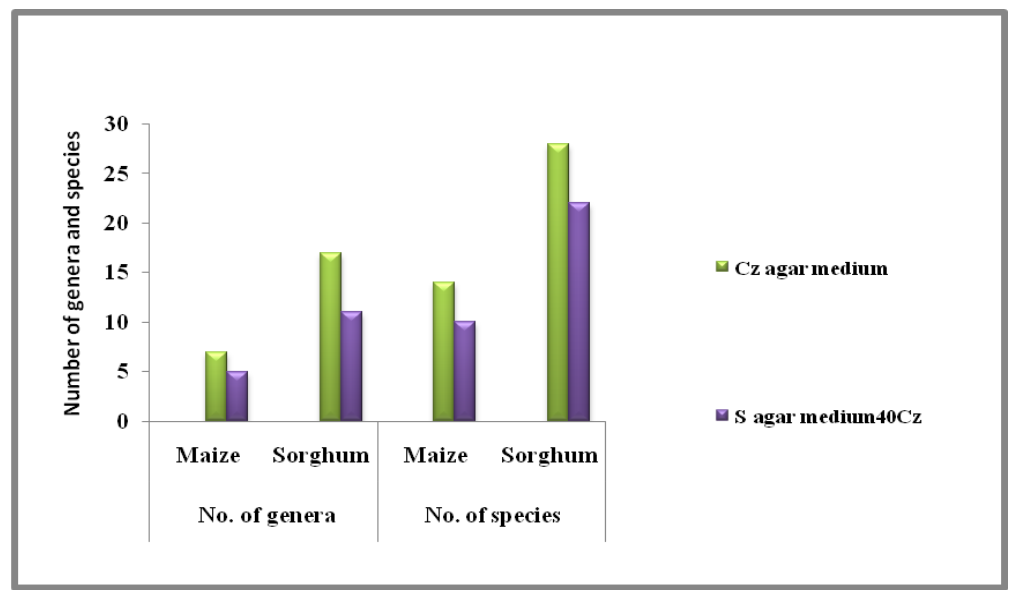

Fig. 2. Diagram illustrating the number of genera (NG) and species (NS) of fungi isolated from maize and sorghum grains (per 100 grains) on $\mathrm{Cz}$ and $\mathrm{Cz} 40 \mathrm{~S}$ agar media.

Egypt. J. Bot. 57, No. 1 (2017) 


\section{Fungi isolated from maize grains}

Seventeen and 11 species related to 7 and 5 genera were recorded from maize grains on $\mathrm{Cz}$ and $\mathrm{Cz} 40 \mathrm{~S}$ agar media, respectively (Table 3). Aspergillus (7 and 6 species; isolated from $95 \%$ and $100 \%$ of total samples, comprising $65.6 \%$ and $55.6 \%$ of total fungi respectively) is the only genus isolated in high occurrence on both $\mathrm{Cz}$ and Cz40S. Whereas, Rhizopus (50\% of total samples and $5.9 \%$ of total fungi), Fusarium (40\% and 16.1\%) and Penicillium (35\% and $8.7 \%$ ) were recorded in high frequency on $\mathrm{Cz}$ and Eurotium (85\% of total samples and $35.9 \%$ of total fungi) was isolated highly on $\mathrm{Cz} 40 \mathrm{~S}$. But Penicillium (30\% and $7.9 \%$ ) was isolated in moderate frequency on $\mathrm{Cz} 40 \mathrm{~S}$. The most common species on both media were A. niger ( $60 \%$ and $85 \%$ of total samples), A. flavus ( $80 \%$ and $65 \%$ ), on $\mathrm{Cz}$ were Rhizopus stolonifer (50\%), F. verticillioides (40\%), Aspergillus tamarii (25\%) and Penicillium pinophilum (30\%), on Cz40S were Eurotium rubrum (45\%), E. repens (40\%), A. ochraceus (30\%), A. tamarii (30\%) and $P$. pinophilum (30\%) (Table 3$)$.

\section{Fungi isolated from sorghum grains}

The results in Table 4 showed that 14 and 10 genera, represented by $27+1$ variety and 22 species were isolated from sorghum grains samples on $\mathrm{Cz}$ and Cz40S agar media, respectively.

Aspergillus was recovered in high frequency (95\% of the total samples on both $\mathrm{Cz}$ and $\mathrm{Cz} 40 \mathrm{~S}$; black sterile mycelia and Rhizopus (50\% each) on $\mathrm{Cz}$ and Eurotium (90\% of total samples) on Cz40S. A. flavus (70\% and $50 \%$ of the total samples), A. niger (55\% and 30\%) and E. amstelodami (30\% and 55\%) were the most common species on both $\mathrm{Cz}$ and $\mathrm{Cz} 40 \mathrm{~S}$, respectively. $R$. stolonifer (50\%) and $F$. verticillioides (30\%) were the most common on $\mathrm{Cz}$ only (Table 4 ).

There are highly significant in total count of Eurotium on both media, while total counts of Penicillium, Fusarium and number of species recorded from maize and sorghum showed high significant difference (Table 2).

\section{Amylase production}

Among the 129 isolates tested, 102 (79.1\% of the total isolates) were able to produce amylase enzyme. Of these isolates, only one isolate related to A. terreus and isolated from sorghum showed high capability $(\mathrm{EI}=1.73)$. On the other hand, 8 isolates were moderate producers, they belonged to Scopulariopsis brevicaulis, C. ovoidea, Fusarium solani, Cochliobolus spicifer (1 isolate each), Drechslera halodes and Penicillium griseofulvum (2 isolates each). The remaining 93 positive isolates were weak producers. The negative producers (27 isolates) were all related to Eurotium group (Table 5). 
TABLE 3. Total counts (TC, calculated per 100 grains in all samples), percentage total counts (TC\%) and percentage frequency (F\%) of fungi isolated from maize grain samples on Czapek's $(\mathrm{Cz})$ and Czapek's supplemented with $40 \%$ sucrose $(\mathrm{Cz40S})$ agar media at $28^{\circ} \mathrm{C}$.

\begin{tabular}{|c|c|c|c|c|c|c|}
\hline \multirow{2}{*}{ Fungal Taxa } & \multicolumn{3}{|c|}{ Czapek's agar } & \multicolumn{3}{|c|}{$\begin{array}{l}\text { Czapek's } 40 \% \\
\text { sucrose agar }\end{array}$} \\
\hline & TC & TC\% & F\% & TC & TC\% & F\% \\
\hline Aspergillus P. Micheli ex Link & 79.25 & 65.6 & 95 & 69.75 & 55.6 & 100 \\
\hline A. flavus Link & 36.5 & 30.2 & 80 & 12 & 9.5 & 65 \\
\hline A. niger van Tieghem & 27 & 22 & 60 & 42.5 & 33.8 & 85 \\
\hline A. ochraceus Wilhelm & 0.25 & 0.2 & 5 & 3.5 & 2.8 & 30 \\
\hline $\begin{array}{l}\text { A. sydowii (Bainier \& Sartory) Thom } \\
\text { \& Church }\end{array}$ & 2.75 & 2.3 & 10 & 2.5 & 1.98 & 5 \\
\hline A. tamarii Kita & 5 & 4.1 & 25 & 7 & 5.6 & 30 \\
\hline A. terreus Thom & 0.75 & 0.62 & 10 & & & \\
\hline $\begin{array}{l}\text { A. vadensis Samson, de Vries, Frisvad } \\
\text { \& Visser }\end{array}$ & 7 & 5.8 & 15 & 2.25 & 1.8 & 10 \\
\hline $\begin{array}{l}\text { Cladosporium cladosporioides } \\
\text { (Fresenius) de Vries }\end{array}$ & 0.25 & 0.2 & 5 & & & \\
\hline $\begin{array}{l}\text { Emericella nidulans (Eidam) } \\
\text { Vuillemin }\end{array}$ & 0.5 & 0.4 & 5 & & & \\
\hline Eurotium Link ex Gray & & & & 45.25 & 35.9 & 85 \\
\hline E. repens de Bary & & & & 27.5 & 21.8 & 40 \\
\hline $\begin{array}{l}\text { E. rubrum Konig, Spieckermann \& } \\
\text { Bremer }\end{array}$ & & & & 17.75 & 14.1 & 45 \\
\hline Fusarium Link & 19.5 & 16.1 & 40 & & & \\
\hline F. proliferatum (Matsush.) Nirenberg & 0.75 & 0.6 & 5 & & & \\
\hline F. solani (Martius) Saccardo & 4.5 & 3.7 & 5 & & & \\
\hline F. verticillioides (Saccardo) Nirenberg & 14.25 & 11.8 & 40 & & & \\
\hline Mucor Fresenius & 1.25 & 1 & 20 & 0.5 & 0.4 & 5 \\
\hline M. circinelloides Tieghem & 0.75 & 0.6 & 15 & & & \\
\hline M. hiemalis Wehmer & 0.5 & 0.4 & 5 & 0.5 & 0.4 & 5 \\
\hline Penicillium Link & 10.5 & 8.7 & 35 & 10 & 7.9 & 30 \\
\hline P. duclauxii Delacroix & 0.25 & 0.2 & 5 & & & \\
\hline P. pinophilum Hedgcock & 10.25 & 8.5 & 30 & 10 & 7.9 & 30 \\
\hline $\begin{array}{l}\text { Rhizopus stolonifer (Ehrenberg) } \\
\text { Vuillemin }\end{array}$ & 7.25 & 5.9 & 50 & 0.25 & 0.2 & 5 \\
\hline Sterile mycelia & 2.5 & 2.1 & 15 & & & \\
\hline Total count & & 121 & & & 126 & \\
\hline No. of genera 8 & & 7 & & & 5 & \\
\hline No. of species 19 & & 17 & & & 11 & \\
\hline
\end{tabular}

Egypt. J. Bot. 57, No. 1 (2017) 
FUNGI ASSOCIATED WITH MAIZE AND SORGHUM GRAINS..

TABLE 4. Total counts (TC, calculated per 100 grains in all samples), percentage total counts $(\mathrm{TC} \%)$ and percentage frequency $(\mathrm{F} \%)$ of fungi isolated from sorghum grain samples on Czapek's $(\mathrm{Cz})$ and Czapek's supplemented with $40 \%$ sucrose $(\mathrm{Cz40S})$ agar media at $28^{\circ} \mathrm{C}$.

\begin{tabular}{|c|c|c|c|c|c|c|}
\hline \multirow[b]{2}{*}{ Fungal Taxa } & \multicolumn{3}{|c|}{ Czapek's agar } & \multicolumn{3}{|c|}{ Czapek's 40\% sucrose agar } \\
\hline & TC & $\mathrm{TC} \%$ & $\begin{array}{l}\mathbf{F} \\
\%\end{array}$ & TC & $\mathrm{TC} \%$ & $\begin{array}{l}\mathbf{F} \\
\%\end{array}$ \\
\hline Alternaria. citri Ellis and Pierce emend., Bliss \& Fawcett & & & & 0.25 & 0.18 & 5 \\
\hline Aspergillus & 89.25 & 64.5 & 95 & 72 & 52.2 & 95 \\
\hline A. aculeatus Lizuka & 9.25 & 6.7 & 25 & 20.75 & 15.03 & 30 \\
\hline A. brasiliensis Varga, Frisvad \& Samson & 6 & 4.3 & 10 & 3.5 & 2.54 & 10 \\
\hline A. clavatus Desmazieres & 8.75 & 6.3 & 20 & & & \\
\hline A. flavus Link & 24.5 & 17.7 & 70 & 12.75 & 9.24 & 50 \\
\hline A. flavus var. columnaris Raper \& Fennell & 2.75 & 1.98 & 10 & & & \\
\hline A. japonicas Saito & & & & 0.25 & 0.18 & 5 \\
\hline A. niger van Tieghem & 33.25 & 24 & 55 & 17 & 12.3 & 30 \\
\hline A. parasiticus Speare & & & & 5 & 3.62 & 5 \\
\hline A. tamarii Kita & 1.75 & 1.3 & 15 & 1.5 & 1.1 & 15 \\
\hline A. terreus Thom & 0.5 & 0.3 & 10 & & & \\
\hline A. vadensis Samson, de Vries, Frisvad \& Visser & 2.5 & 1.8 & 5 & 11.25 & 8.15 & 25 \\
\hline Chaetomium globosum Kunze & 0.5 & 0.3 & 5 & & & \\
\hline Cladosporium Link & 0.25 & 0.2 & 5 & 1.25 & 0.9 & 15 \\
\hline C. cladosporioides (Fresenius) de Vries & 0.25 & 0.2 & 5 & 0.5 & 0.36 & 10 \\
\hline C. sphaerospermum Penzig & & & & 0.75 & 0.5 & 5 \\
\hline Cochliobolus spicifer Nelson & 0.5 & 0.3 & 10 & & & \\
\hline Curvularia Boedijn & 1.5 & 1.1 & 20 & & & \\
\hline C. lunata (Wakker) Boedijn & 1.25 & 0.9 & 15 & & & \\
\hline C. ovoidea (Hiroe \&Watan.) Muntanole & 0.25 & 0.2 & 5 & & & \\
\hline Drechslera halodes (Drechsler) Subram and Jain & 2.5 & 1.8 & 25 & 0.25 & 0.18 & 5 \\
\hline Emericella nidulans (Eidam) Vuillemin & & & & 0.25 & 0.18 & 5 \\
\hline Eurotium Link ex Gray & 7.25 & 5.3 & 40 & 58.25 & 42.2 & 90 \\
\hline E. amstelodami Mangin & 3.75 & 2.7 & 30 & 20.5 & 14.9 & 55 \\
\hline E. intermedium Blaser & & & & 1.25 & 0.9 & 5 \\
\hline E. pseudoglaucum Blochwitz & & & & 6.25 & 4.53 & 15 \\
\hline E. repens de Bary & & & & 11.75 & 8.51 & 30 \\
\hline E. rubrum Konig, Spieckermann \& Bremer & 3.5 & 2.5 & 10 & 18.5 & 13.4 & 35 \\
\hline Fusarium Link & 5.75 & 4.2 & 35 & 0.25 & 0.18 & 5 \\
\hline F. lateritium Nees & 1 & 0.7 & 10 & & & \\
\hline F. verticillioides (Saccardo) Nirenberg & 4.75 & 3.4 & 30 & 0.25 & 0.18 & 5 \\
\hline Mucor Fresenius & 4.5 & 3.3 & 25 & 0.25 & 0.18 & 5 \\
\hline M. circinelloides Tieghem & 4 & 2.9 & 25 & 0.25 & 0.18 & 5 \\
\hline M. racemosus Fresenius & 0.5 & 0.3 & 10 & & & \\
\hline Penicillium Link & 1.5 & 1.1 & 15 & 0.75 & 0.5 & 15 \\
\hline P. duclauxii Delacroix & 0.25 & 0.2 & 5 & & & \\
\hline P. griseofulvum Dierckx & 1.25 & 0.9 & 10 & 0.75 & 0.5 & 15 \\
\hline Phoma Saccardo & 1.5 & 1.1 & 25 & & & \\
\hline P. glomerata (Corda) Wollenw \& Hochapfel & 0.25 & 0.2 & 5 & & & \\
\hline P. herbarum Westendorp & 1.25 & 0.9 & 20 & & & \\
\hline Rhizopus stolonifer (Ehrenberg) Vuillemin & 8.25 & 6 & 50 & 1.25 & 0.9 & 20 \\
\hline Scopulariopsis brevicaulis(Saccardo) Bainier & 0.25 & 0.2 & 5 & & & \\
\hline Sterile mycelia (black) & 14.25 & 10.2 & 50 & 3.25 & 2.4 & 20 \\
\hline Trichoderma longibrachiatum Rifai & 0.25 & 0.2 & 5 & & & \\
\hline Yeast & 0.25 & 0.2 & 5 & & & \\
\hline Total count & & 139 & & & 138 & \\
\hline No. of genera 16 & & 14 & & & 10 & \\
\hline No. of species $(35+1$ variety) & & -1 variet & & & 22 & \\
\hline
\end{tabular}

Egypt. J. Bot.57, No. 1 (2017) 
TABLE 5. $\alpha$ - amylase enzyme production by the most commonly fungi recovered from maize and sorghum grains.

\begin{tabular}{|c|c|c|c|c|c|}
\hline Species & $\begin{array}{c}\text { Isolates } \\
\text { tested }\end{array}$ & Source & $\begin{array}{l}\text { Isolation } \\
\text { medium }\end{array}$ & $\begin{array}{l}\text { Enzyme } \\
\text { index }\end{array}$ & OR \\
\hline Aspergillus terreus & 1 & Sorghum & $\mathrm{Cz}$ & 1.73 & $\mathrm{H}$ \\
\hline Scopulariopsis brevicaulis & 1 & Sorghum & $\mathrm{Cz}$ & 1.5 & $\mathrm{M}$ \\
\hline Curvularia ovoidea & 1 & Sorghum & $\mathrm{Cz}$ & 1.4 & $\mathrm{M}$ \\
\hline Fusarium solani & 1 & Maize & $\mathrm{Cz}$ & 1.4 & $\mathrm{M}$ \\
\hline Cochliobolus spicifer & 1 & Sorghum & $\mathrm{Cz}$ & 1.3 & $M$ \\
\hline \multirow{2}{*}{ Drechslera halodes } & \multirow[t]{2}{*}{2} & Maize & $\mathrm{Cz}$ & 1.3 & $\mathrm{M}$ \\
\hline & & Sorghum & $\mathrm{Cz}$ & 1.3 & $\mathrm{M}$ \\
\hline Penicillium griseofulvum & 2 & Sorghum & $\mathrm{Cz}$ & 1.3 & $\mathrm{M}$ \\
\hline Alternaria citri & 2 & Sorghum & $\mathrm{Cz} 40 \mathrm{~S}$ & 1 & $\mathrm{~W}$ \\
\hline Aspergillus aculeatus & 4 & Sorghum & $\mathrm{Cz}$ & $1.04-1.1$ & $\mathrm{~W}$ \\
\hline A. brasiliensis & 5 & Sorghum & $\mathrm{Cz}$ and $\mathrm{Cz} 40 \mathrm{~S}$ & $1.03-1.1$ & $\mathrm{~W}$ \\
\hline A. clavatus & 1 & Sorghum & $\mathrm{Cz}$ & 1.03 & $\mathrm{~W}$ \\
\hline A. flavus & $\begin{array}{l}6 \\
5\end{array}$ & $\begin{array}{l}\text { Maize } \\
\text { Sorghum }\end{array}$ & $\begin{array}{l}\mathrm{Cz} \text { and } \mathrm{Cz} 40 \mathrm{~S} \\
\mathrm{Cz} \text { and } \mathrm{Cz} 40 \mathrm{~S}\end{array}$ & $\begin{array}{l}1-1.2 \\
1.02-1.2\end{array}$ & $\begin{array}{l}\mathrm{W} \\
\mathrm{W}\end{array}$ \\
\hline $\begin{array}{l}\text { A. flavus var. columnaris } \\
\text { A. japonicas }\end{array}$ & $\begin{array}{l}2 \\
1\end{array}$ & $\begin{array}{l}\text { Sorghum } \\
\text { Sorghum }\end{array}$ & $\begin{array}{l}\mathrm{Cz} \\
\mathrm{Cz} 40 \mathrm{~S}\end{array}$ & $\begin{array}{l}1.1 \\
1.03 \\
\end{array}$ & $\begin{array}{l}\mathrm{W} \\
\mathrm{W}\end{array}$ \\
\hline A. niger & $\begin{array}{c}7 \\
10\end{array}$ & $\begin{array}{l}\text { Maize } \\
\text { Sorghum }\end{array}$ & $\begin{array}{l}\mathrm{Cz} \text { and } \mathrm{Cz} 40 \mathrm{~S} \\
\mathrm{Cz} \text { and } \mathrm{Cz} 40 \mathrm{~S}\end{array}$ & $\begin{array}{l}1-1.1 \\
1.03-1.1\end{array}$ & $\begin{array}{l}\mathrm{W} \\
\mathrm{W}\end{array}$ \\
\hline $\begin{array}{l}\text { A. ochraceus } \\
\text { A. sydowii }\end{array}$ & $\begin{array}{l}4 \\
2\end{array}$ & $\begin{array}{l}\text { Maize } \\
\text { Maize }\end{array}$ & $\begin{array}{l}\mathrm{Cz} 40 \mathrm{~S} \\
\mathrm{Cz} 40 \mathrm{~S}\end{array}$ & $\begin{array}{l}1.15-1.2 \\
1.15-1.2\end{array}$ & $\begin{array}{l}\mathrm{W} \\
\mathrm{W}\end{array}$ \\
\hline A. tamarii & $\begin{array}{l}2 \\
1 \\
\end{array}$ & $\begin{array}{l}\text { Maize } \\
\text { Sorghum }\end{array}$ & $\begin{array}{l}\mathrm{Cz} \text { and } \mathrm{Cz} 40 \mathrm{~S} \\
\mathrm{Cz}\end{array}$ & $\begin{array}{l}1.1 \\
1.2 \\
\end{array}$ & $\begin{array}{l}\mathrm{W} \\
\mathrm{W}\end{array}$ \\
\hline $\begin{array}{l}\text { A. vadensis } \\
\text { Chaetomium globosum }\end{array}$ & $\begin{array}{l}1 \\
1\end{array}$ & $\begin{array}{l}\text { Sorghum } \\
\text { Sorghum }\end{array}$ & $\begin{array}{l}\mathrm{Cz} 40 \mathrm{~S} \\
\mathrm{Cz} 40 \mathrm{~S}\end{array}$ & $\begin{array}{l}1.1 \\
1\end{array}$ & $\begin{array}{l}\mathrm{W} \\
\mathrm{W}\end{array}$ \\
\hline Cladosporium cladosporioides & 2 & Sorghum & $\mathrm{Cz}$ & 1 & $\mathrm{~W}$ \\
\hline $\begin{array}{l}\text { C. sphaerospermum } \\
\text { Cochliobolus spicifer }\end{array}$ & $\begin{array}{l}1 \\
2\end{array}$ & $\begin{array}{l}\text { Sorghum } \\
\text { Sorghum }\end{array}$ & $\begin{array}{l}\mathrm{Cz} \\
\mathrm{Cz} \text { and } \mathrm{Cz} 40 \mathrm{~S}\end{array}$ & $\begin{array}{l}1 \\
1.05 \\
\end{array}$ & $\begin{array}{l}\mathrm{W} \\
\mathrm{W}\end{array}$ \\
\hline $\begin{array}{l}\text { Curvularia lunata } \\
\text { C. ovoidea }\end{array}$ & $\begin{array}{l}6 \\
2 \\
\end{array}$ & $\begin{array}{l}\text { Sorghum } \\
\text { Sorghum }\end{array}$ & $\begin{array}{l}\mathrm{Cz} \text { and } \mathrm{Cz} 40 \mathrm{~S} \\
\mathrm{Cz} \text { and } \mathrm{Cz} 40 \mathrm{~S}\end{array}$ & $\begin{array}{l}1-1.15 \\
1-1.05 \\
\end{array}$ & $\begin{array}{l}\mathrm{W} \\
\mathrm{W}\end{array}$ \\
\hline Drechslera halodes & $\begin{array}{l}1 \\
3\end{array}$ & $\begin{array}{l}\text { Maize } \\
\text { Sorghum }\end{array}$ & $\begin{array}{l}\mathrm{Cz} \\
\mathrm{Cz}\end{array}$ & $\begin{array}{l}1.2 \\
1-1.1\end{array}$ & $\begin{array}{l}\mathrm{W} \\
\mathrm{W}\end{array}$ \\
\hline $\begin{array}{l}\text { Fusarium lateritium } \\
\text { F. solani }\end{array}$ & $\begin{array}{l}2 \\
2\end{array}$ & $\begin{array}{l}\text { Sorghum } \\
\text { Maize }\end{array}$ & $\begin{array}{l}\mathrm{Cz} \text { and } \mathrm{Cz} 40 \mathrm{~S} \\
\mathrm{Cz}\end{array}$ & $\begin{array}{l}1.15-1.2 \\
1\end{array}$ & $\begin{array}{l}\mathrm{W} \\
\mathrm{W}\end{array}$ \\
\hline F. verticillioides & $\begin{array}{l}3 \\
2\end{array}$ & $\begin{array}{l}\text { Maize } \\
\text { Sorghum }\end{array}$ & $\begin{array}{l}\mathrm{Cz} \\
\mathrm{Cz}\end{array}$ & $\begin{array}{l}1-1.2 \\
1\end{array}$ & $\begin{array}{l}\text { W } \\
\text { W }\end{array}$ \\
\hline $\begin{array}{l}\text { Mucor circinelloides } \\
\text { Penicillium duclauxii }\end{array}$ & $\begin{array}{l}1 \\
1\end{array}$ & $\begin{array}{l}\text { Sorghum } \\
\text { Sorghum }\end{array}$ & $\begin{array}{l}\mathrm{Cz} \\
\mathrm{Cz}\end{array}$ & $\begin{array}{l}1.1 \\
1\end{array}$ & $\begin{array}{l}\mathrm{W} \\
\mathrm{W}\end{array}$ \\
\hline \multirow{2}{*}{ P. griseofulvum } & 1 & Maize & $\mathrm{Cz}$ & 1.15 & $\mathrm{~W}$ \\
\hline & 3 & Sorghum & $\mathrm{Cz}$ and $\mathrm{Cz} 40 \mathrm{~S}$ & $1.04-1.2$ & $\mathrm{~W}$ \\
\hline P. pinophilum & 3 & Maize & $\mathrm{Cz}$ and $\mathrm{Cz} 40 \mathrm{~S}$ & $1-1.1$ & $\mathrm{~W}$ \\
\hline Phoma glomerata & 2 & Sorghum & $\mathrm{Cz}$ & 1 & $\mathrm{~W}$ \\
\hline P. herbarum & 1 & Sorghum & $\mathrm{Cz}$ & 1 & $\mathrm{~W}$ \\
\hline Trichoderma longibrachiatum & 1 & Sorghum & $\mathrm{Cz}$ & 1 & $\mathrm{~W}$ \\
\hline \multirow{2}{*}{ Eurotium amstelodami } & 3 & Maize & $\mathrm{Cz} 40 \mathrm{~S}$ & $-\mathrm{Ve}$ & $-\mathrm{Ve}$ \\
\hline & 6 & Sorghum & $\mathrm{Cz}$ and $\mathrm{Cz} 40 \mathrm{~S}$ & $-\mathrm{Ve}$ & $-\mathrm{Ve}$ \\
\hline E. intermedium & 4 & Sorghum & $\mathrm{Cz}$ and $\mathrm{Cz} 40 \mathrm{~S}$ & $-\mathrm{Ve}$ & $-\mathrm{Ve}$ \\
\hline E. pseudoglaucum & 1 & Sorghum & $\mathrm{Cz}$ and $\mathrm{Cz} 40 \mathrm{~S}$ & $-\mathrm{Ve}$ & $-\mathrm{Ve}$ \\
\hline \multirow{2}{*}{ E. repens } & 5 & Maize & $\mathrm{Cz} 40 \mathrm{~S}$ & $-\mathrm{Ve}$ & $-\mathrm{Ve}$ \\
\hline & 4 & Sorghum & $\mathrm{Cz}$ and $\mathrm{Cz} 40 \mathrm{~S}$ & $-\mathrm{Ve}$ & $-\mathrm{Ve}$ \\
\hline E. rubrum & 4 & Sorghum & $\mathrm{Cz}$ and $\mathrm{Cz} 40 \mathrm{~S}$ & $-\mathrm{Ve}$ & $-\mathrm{Ve}$ \\
\hline
\end{tabular}

$\mathrm{H}=$ High production $(\mathrm{EI}=1.6-1.7)$

$\mathrm{M}=$ Moderate production $(\mathrm{EI}=1.3-1.5)$

$\mathrm{W}=$ Weak production $(\mathrm{EI}=1-1.2)$

Egypt. J. Bot. 57, No. 1 (2017) 
Fluorescence (at $365 \mathrm{~nm}$ ) of Aspergillus flavus group on Coconut agar medium (CAM)

Nineteen isolates of Section Flavi, including A. flavus (17 isolates), A. flavus var. columnaris and $A$. tamarii (one isolate each) collected from maize and sorghum grains were screened for their abilities to produce aflatoxin on CAM. The results revealed that 7 isolates $(36.8 \%$ of total isolates) were able to produce fluorescence under U.V light at $365 \mathrm{~nm}$, this indicates the production of aflatoxin. A. flavus strain No. AUMC 11314 gave very high fluorescence $(++++)$, while strain Nos. AUMC 11311, AUMC 11312, AUMC 11313 and No. AUMC 11317 showed high aflatoxin production (+++) (Table 6).

TABLE 6. Fluorescence (at $365 \mathrm{~nm}$ ) of Aspergillus flavus group recovered from maize and sorghum grains as revealed on coconut agar medium (CAM).

\begin{tabular}{|c|c|c|c|c|}
\hline \multirow[b]{2}{*}{ Species } & \multicolumn{2}{|c|}{ Maize } & \multicolumn{2}{|c|}{ Sorghum } \\
\hline & Strain No. & $\begin{array}{c}\text { Fluorescence } \\
\text { on CAM }\end{array}$ & AUMC No. & $\begin{array}{c}\text { Fluorescence } \\
\text { on CAM }\end{array}$ \\
\hline Aspergillus flavus & 11325AUMC & -ve & AUMC 11321 & -ve \\
\hline A. flavus & 11326AUMC & -ve & 11311 AUMC & +++ \\
\hline A. flavus & 11327AUMC & -ve & 11322 AUMC & -ve \\
\hline A. flavus & $\begin{array}{c}\text { AUMC } \\
11314 \\
\end{array}$ & ++++ & AUMC 11323 & -ve \\
\hline A. flavus & $\begin{array}{c}\text { AUMC } \\
11315 \\
\end{array}$ & ++ & AUMC 11312 & +++ \\
\hline A. flavus & $\begin{array}{c}\text { AUMC } \\
11328 \\
\end{array}$ & -ve & AUMC 11324 & -ve \\
\hline A. flavus & $\begin{array}{l}11316 \\
\text { AUMC }\end{array}$ & + & AUMC 11313 & +++ \\
\hline A. flavus & $\begin{array}{c}\text { AUMC } \\
11329 \\
\end{array}$ & -ve & & \\
\hline A. flavus & $\begin{array}{l}11330 \\
\text { AUMC }\end{array}$ & -ve & & \\
\hline A. flavus & $\begin{array}{c}\text { AUMC } \\
11317 \\
\end{array}$ & +++ & & \\
\hline A. flavus var. columnaris & & & 11320 AUMC & -ve \\
\hline A. tamarii & $\begin{array}{c}11331 \\
\text { AUMC }\end{array}$ & -ve & & \\
\hline
\end{tabular}

fluorescence on CAM is expressed as -ve: negative result, +: weak intensity, ++: moderate intensity, +++ : high intensity and ++++: very high intensity.

Assessment of mycotoxins by HPLC.

Using high performance liquid chromatography (HPLC), 6 strains of $A$. flavus collected from maize and sorghum grains (3 isolates each) showed production of aflatoxins $B_{1}, B_{2}$ and $G_{2}$ with various degrees. Strain No. AUMC 11311 produced the highest value of both aflatoxins $B_{1}$ and $B_{2}(0.733 \mathrm{mg} / \mathrm{l}$ and 
$0.034 \mathrm{mg} / \mathrm{l})$, while, No. AUMC 11317 had the highest value of aflatoxin $\mathrm{G}_{2}$ $(0.871 \mathrm{mg} / \mathrm{l})$ (Table 7$)$.

TABLE 7. Mycotoxins screening potential of different isolates isolated from maize and sorghum by HPLC.

\begin{tabular}{|c|c|c|c|c|c|}
\hline \multirow{2}{*}{ Species } & \multirow{2}{*}{ No. Strain } & \multirow{2}{*}{ Source } & \multicolumn{3}{|c|}{ Aflatoxin } \\
\hline & & & $\mathbf{B}_{1}$ & $\mathbf{B}_{2}$ & $\mathbf{G}_{2}$ \\
\hline Aspergillus flavus & 11314 AUMC & Maize & $0.007 \mathrm{mg} / \mathrm{l}$ & $0.016 \mathrm{mg} / 1$ & $0.553 \mathrm{mg} / \mathrm{l}$ \\
\hline A. flavus & 11315 AUMC & Maize & $0.003 \mathrm{mg} / 1$ & $0.003 \mathrm{mg} / 1$ & $0.613 \mathrm{mg} / 1$ \\
\hline A. flavus & 11317 AUMC & Maize & $0.002 \mathrm{mg} / \mathrm{l}$ & $0.002 \mathrm{mg} / \mathrm{l}$ & $0.871 \mathrm{mg} / \mathrm{l}$ \\
\hline A. flavus & 11311 AUMC & Sorghum & $0.733 \mathrm{mg} / \mathrm{l}$ & $0.034 \mathrm{mg} / \mathrm{l}$ & $0.442 \mathrm{mg} / 1$ \\
\hline A. flavus & 11312 AUMC & Sorghum & $0.024 \mathrm{mg} / \mathrm{l}$ & $0.013 \mathrm{mg} / \mathrm{l}$ & $0.714 \mathrm{mg} / \mathrm{l}$ \\
\hline A. flavus & 11313 AUMC & Sorghum & $0.006 \mathrm{mg} / 1$ & $0.004 \mathrm{mg} / \mathrm{l}$ & $0.585 \mathrm{mg} / 1$ \\
\hline
\end{tabular}

\section{Discussion}

Knowledge of the composition of mycobiota in cereal grains, during or postharvest and in storage is an important step towards the prediction of possible mycotoxin contamination, accordingly, avoiding harmful effect on yield and grain quality.

Moisture content of the grain is a critical factor for fungal growth on the grain, leading to quality loss. Moisture contents of sorghum grain samples were much higher in sorghum than in maize. Moisture content is one of several factors known to influence fungal development and secondary metabolite production in agricultural products (Ezekiel et al., 2014). Also, it has important role in enzyme production by fungi (Dar et. al., 2014). It was reported that, storage fungi require moisture content ranging between $13-18 \%$ to invade cereal starchy grains (Moubasher et al., 1972).

The germinability of grains was slightly higher in maize (40-100\%) than in sorghum (30-90\%), this may be due to high moisture contents in sorghum grains (up to $20.2 \%$ ) which stimulate fungal growth. In agreement with the current results, Moubasher et al. (1980) stated that the germinability of peanut seeds declined with raising the moisture content.

Fungi isolated from maize grains

A total of 19 species belonging to 8 genera were collected from maize grains. Aspergillus, Eurotium, Fusarium and Rhizopus were the most common genera of which A. flavus, A. niger, E. rubrum, E. repens, Rhizopus stolonifer and $F$. verticillioides were the most encountered. El-Shanshoury et al. (2014) found that A. flavus, A. niger, Penicillium spp. and Fusarium spp. were the most common fungi in samples of cereal grains (maize and wheat) and peanut collected from central Delta province, Egypt. Also, Abe et al. (2015) recorded Fusarium and Aspergillus as the highest diversity fungi in maize grains of Brazil. Most fungi

Egypt. J. Bot. 57, No. 1 (2017) 
isolated in the current study (e.g. Aspergillus, Cladosporium, Curvularia, Fusarium, Mucor, Penicillium and Trichoderma) were frequently found in maize from Pakistan, Saudi Arabia and Ethiopia (Niaz and Dawar, 2009, Mohamed et al., 2013 and Ofgea and Gure, 2015).

\section{Fungi isolated from sorghum grains}

The current results showed that, number of genera (16) and species (35), as well as, total counts of fungi isolated from sorghum grains were relatively higher than those isolated from maize ( 8 genera, 19 species), this means that the higher moisture contents of sorghum (up to 20.2\%) than those of maize grains (up to $11.2 \%$ ) enhance fungal diversity and population on sorghum. Aspergillus, Eurotium, Fusarium, Rhizopus and black sterile mycelia were the most frequent genera. A. flavus, A. niger, Rhizopus stolonifer, E. amstelodami and $F$. verticillioides were the most common species.

A similar trend was reported for stored sorghum grains in Kenya (Kange et al., 2015). In Nigeria, Abdulsalaam and Shenge (2011) recorded Aspergillus, Fusarium, Rhizoctonia and Curvularia species as the most common in washed sorghum grains. In a study of Ismail et al. (2012), Aspergillus and Eurotium were isolated in high incidences from cereal baby foods locally produced in Uganda.

amylase production $-\alpha$

The highly production of $\alpha$ - amylase by to A. terreus isolated from sorghum. In the current work is supported by the studies of Khairnar (2014) and Ogbonna et al. (2015) who found that strains of A. niger and A. terreus were highly amylase producers. In accordance with our results, A. niger, A. flavus, A. japonicus, A. terreus, Cladosporium cladosporioides and Chaetomium globosum were also capable of producing amylase with the strains of $A$. niger and $C$. cladosporioides are the best producers (Reddy and Sreeramulu, 2016). Our finding on the lack of amylase production by the xerophilic species of Eurotium, disagree with those of Ulfig et al. (2009) and Shivani and Kumar (2015). The above mentioned fungi were previously recorded as $\alpha$ - amylase producers from various substrates (Dar et al., 2014, Pathak et al., 2014 and Lall et al., 2015).

\section{Aflatoxin production}

The current results revealed that $36.8 \%$ of total isolates tested (19) were able to produce aflatoxins. In this respect, Ismail et al. (2016) stated after screening 47 isolates collected from peanut, corn and wheat on CAM that, two A. flavus strains (from corn) and 5 (from wheat) showed intense blue fluorescence indicating very high production of aflatoxin $\mathrm{B}$. While 12 strains from peanut and 5 from wheat were high producers. Several studies reported the aflatoxigenic potential of different $A$. flavus strains collected from various seeds and grains all over the world (Riba et al., 2010, Ezekiel et al., 2012, Kana et al., 2013 and Fakruddin et al., 2015). 
The results obtained by HPLC showed that, 6 strains of A. flavus could produce aflatoxins $B_{1}, B_{2}$ and $G_{2}$, but strains No. AUMC 11311 (for aflatoxins $\mathrm{B}_{1}$ and $\mathrm{B}_{2}$ ) and No. AUMC 11317 (for aflatoxin $\mathrm{G}_{2}$ ) were the highest producers. Ezekiel et al. (2014) observed that, toxigenic strain of A. flavus obtained from fonio millet produced higher amounts of aflatoxin B than those from sesame. Moreover, the level of aflatoxin $G$ was higher than that of aflatoxin B. In accordance with the current results, Aflatoxins $B_{1}, B_{2}, G_{1}$ and $G_{2}$ produced previously by several isolates of $A$. flavus collected from various substrates (ElMaraghy and Zohri, 1988, Feizy et al., 2012, Abu-Taleb et al., 2012, Kange et al. 2015, Lai et al., 2015 and Hamed et al., 2016).

\section{Conclusion}

The incidence of moulds and levels of mycotoxins in foods should be frequently and routinely determined. Also, there is an urgent need for further studies on fungi associated with stored cereal grains and their enzymes and mycotoxin production.

\section{References}

Abdel-Hafez, S.I.I., Ismail, M.A., Hussein, N.A. and Abdel-Hameed, N.A. (2014) Fusarium species and other fungi associated with some seeds and grains in Egypt, with 2 newly recorded Fusarium species. J. Biol. Earth Sci. 4 (2),120-129.

Abdulsalaam, S. and Shenge, K.C. (2011) Seed borne pathogens on farmer-saved sorghum (Sorghum bicolor L.) seeds. J. Stored Prod. Postharvest Res. 2 (2), 24-28.

Abe, C.A.L., Faria, C.B., Castro, F.F., Souza, S.R., Santos, F.C., Silva, C.N., Tessmann, D.J. and Barbosa, I.P. (2015) Fungi isolated from maize (Zea mays L.) grains and production of associated enzyme activities. Int. J. Mol. Sci., 16 (7),1532815346 .

Abu-Taleb, A.M., Al -Julif, M.Z. and Al -Arjani, A.F. (2012) Toxigenic fungi isolated from some food commodities and their phytotoxicity. Egypt. J. Exp. Biol., (Bot.) 8 (1), $141-149$.

Booth, C. (1971) "The Genus Fusarium". Commonwealth Mycological Institute, Kew, Surrey, England $237 \mathrm{pp}$.

Bridge, P.D. (1985) An evaluation of some physiological and biochemical methods as an aid to the characterization of species of Penicillium subsection fasciculata. J. Gen. Microbiol, 131, 1887-1895.

Cowan, S.T. (1974) Cowan and Steel's Manual for the Identification of Medical Bacteria, $2^{\text {nd }}$ ed. Cambridge University Press, Cambridge pp.250.

Dar, G.H., Kamil, A.N., Nazir, R., Bandh, S.A. and Malik, T.A. (2014) Biotechnology production of $\alpha$ - amylase for industrial purpose: Do fungi have potential to produce $\alpha$ amylase? Int. J. Biotechnol. Mol. Biol. Res., 5 (4), 35-40. 
Davis, N.D., Iyer, S.K. and Diener, U.L. (1987) Improved method of screening for aflatoxin with a coconut agar medium. Applied and Environmental Microbiology 53 (7), 1593-1595.

Domsch, K.H., Gams, W. and Anderson, T.H. (2007) "Compendium of Soil Fungi". $2^{\text {nd }}$ edition, IHC-Verlag, Eching pp 672.

Ellis, M.B. (1971) "Dematiaceous Hyphomycetes". "Commonwealth Mycological Institute, Kew, Surrey, England, pp 608.

El-Maraghy, S.S. and Zohri, A.A. (1988) Mycotoxin-producing potential of aspergilli and penicillia of broad beans in Egypt. Bulletin Faculty of Science, Assiut University, Egypt, 17 (1), 91-102.

El-Shanshoury, A.R., El-Sabbagh, S.M., Emara, H.A. and Saba, H.E. (2014) Occurrence of moulds, toxicogenic capability of Aspergillus flavus and levels of aflatoxins in maize, wheat, rice and peanut from markets in central delta Provinces, Egypt. Int. J. Curr. Microbiol. App. Sci., 3 (3), 852-865.

Ezekiel, C.N., Kayode, F.O., Fapohunda, S.O., Olorunfemi, M.F. and Kponi B.T. (2012) Aflatoxigenic moulds and aflatoxins in street-vended snacks in Lagos, Nigeria. Internet Journal of Food Safety, 14, 83-88.

Ezekiel, C.N., Udom, I.E., Frisvad, J.C., Adetunji, M.C., Houbraken, J., Fapohunda, S.O., Samson, R.A., Atanda, O.O., Agi-Otto, M.C. and Onashile, O.A. (2014) Assessment of aflatoxigenic Aspergillus and other fungi in millet and sesame from Plateau State, Nigeria. Mycology, 5 (1), 16-22.

Fakruddin, M., Chowdhury A., Hossain, M.N. and Ahmed M.M. (2015) Characterization of aflatoxin producing Aspergillus flavus from food and feed samples. Springerplus, 4 (159), 1-6.

Feizy, J., Beheshti, H.R. and Asadi, M. (2012) Ochratoxin A and aflatoxins in dried vine fruits from the Iranian market. Mycotoxin Research , 28 (4), 237-242.

Gummert, M. (2011) Measuring seed germination. In: "Postharvest Fact Sheets". IRRI, Manila.

Hamed, M.A., Abdel Ghany, T.M, Elhussieny, N.I. and Nabih, M.A. (2016) Exploration of fungal infection in agricultural grains, aflatoxin and zearalenone synthesis under pH stress. Int. J. Curr. Microbiol. App. Sci., 5 (4), 1007-1017.

Ho, H.H. and Foster, B. (1972) Starch utilization by Phytophthora species. Mycopathol. Mycol. Appl., 46 (4), 335-339.

(IARC) International Agency for Research on Cancer (2002) Some traditional herbal medicines, some mycotoxins, naphthalene and styrene. IARC monographs on the evaluation of carcinogenic risks to Humans Lyon, France : World Health Organization, 82, 1-556. 
Irfan, M., Nadeem, M. and Syed, Q. (2012) Media optimization for amylase production in solid state fermentation of wheat bran by fungal strains. J. Cell. Mol. Biol. 10 (1), $55-64$.

Ismail, M.A., Taligoola, H.K. and Nakamya, R. (2012) Xerophiles and other fungi associated with cereal baby foods locally produced in Uganda. Acta Mycologica, 47(1), 75-89.

Ismail, M.A., Abdel- Hafez, S.I.I., Hussein, N.A. and Abdel- Hameed, N.A. (2015) "Contributions to the Genus Fusarium in Egypt with Dichotomous Keys for Identification of Species”. ul. Szkółkarska 88B, 62-002 Suchy Las, Poland, 175 pp.

Ismail, M.A., Abo El-Maali, N.T., Omran, G.A. and Nasser, M.N. (2016) Biodiversity of mycobiota in peanut seeds, corn and wheat grains with special reference to their aflatoxigenic ability. J . Microbiol. Biotech. Food Sci., 5 (4), 314-319.

Kana, J.R., Gnonlonfin, B.G.J., Harvey, J., Wainaina, J., Wanjuki, I., Skilton, R. A., Teguia, A. (2013) Assessment of aflatoxin contamination of maize, peanut meal and poultry feed mixtures from different agroecological zones in Cameroon. Toxins 5 (5), 884-894.

Kange, A.M., Cheruiyot, E.K., Ogendo, J.O. and Arama, P.F. (2015) Effect of sorghum (Sorghum bicolor L. Moench) grain conditions on occurrence of mycotoxinproducing fungi. Agric \& Food Secur.15 (4), 2-8.

Khairnar, D.N. (2014) Studies on diversity, amylase production by seed- borne fungi of pearl millet and their control measures. Int. Res. J. of Science \& Engineering 2 (2), 68-70.

Lai, X., Zhang, H., Liu, R. and Liu, C. (2015) Potential for aflatoxin B1 and B2 production by Aspergillus flavus strains isolated from rice samples. Saudi Journal of Biological Sciences, 22 (2), 176-180.

Lall, B.M., Paul, J.S. and Jadhav, S.K. (2015) Effect of incubation period (with static and shaking conditions)on $\alpha$ - amylase production from Aspergillus flavus. Advan. Biol. Res. 9 (1), 1-6.

Leslie, J.F. and Summerrell, B.A. (2006) "The Fusarium: Laboratory Manual". Blackwell Publishing, 388 pp.

Machio, K.A. (2016) Mycoflora compositions of sorghum (Sorghum bicolor L. Moench) grains from eastern region of Kenya. JAERI 8 (2), 1-13.

Mohamed, A.M., Monira, R.A. and Abeer R.M.A. (2013) Mycotoxigenic fungi contaminating corn and sorghum grains in Saudi Arabia. P.J. Bot. 45 (5), 1831-1839.

Mohammed, K., Gure A. and Zuberi, M.I. (2015) Problems of seed-borne fungal diseases affecting sorghum grain (Sorghum bicolor L. Moench) in two districts of Oromia, Ethiopia. International Journal of Biosciences 7 (5), 66-77.

Moubasher, A.H. (1993) "Soil Fungi of Qatar and other Arab Countries". The Scientific and Applied Research Centre, University of Qatar, Doha, Qatar, 566 pp.

Egypt. J. Bot. 57, No. 1 (2017) 
Moubasher, A.H., Elnaghy, M.A. and Abdel-Hafez S.I.I. (1972) Studies on the fungus flora of three grains in Egypt. Mycopathol. Mycol. Appl. 47 (3), 261-274.

Moubasher, A.H., Abdel-Hafez, S.I.I., El-Hissy, F.T. and Hassan, S.K.M. (1980) Effect of temperature and moisture content on Egyptian peanut seed-borne fungi. Mycopathologia., 70 (1), 49-54.

Niaz, I. and Dawar, S. (2009) Detection of seed borne mycoflora in Maize (Zea mays L.). P. J. Bot. 41 (1), 443-451.

Ofgea, K.C. and Gure A. (2015) Morphological diversity of fungi associated with stored grains of maize (Zea mays L.) in Shashemene and Arsi Nagelle districts, Ethiopia. I J I. S. R.15 (1), 142-149.

Ogbonna, A.I., Onyimba, I.A., Chuku, A., Nwadiaro, P.O., Ogbonna, C.I.C. and Onwuliri, F.C. (2015) Growth response and amylolytic activity of two Aspergillus species isolated from Artemisia annua L. plantation soils. Eur. J. Biotechnol Bioscience., 3 (10), 10- 16.

Pathak, S.S., Kumar, S., Rajak, R.C. and Sandhu, S.S. (2014) Study of effect of temperature on amylase production by soil mycotic flora of jabalpur region. World $J$. Pharm. Pharmaceut Sci., 3 (9), 1448-1458.

Pitt, J.I. (1979) "The Genus Penicillium and its Teleomorphic States Eupenicillium and Talaromyces.” Academic Press, London ,634 pp.

Pitt, J.I. and Hocking, A.D. (2009) "Fungi and Food Spoilage". $3^{\text {rd }}$ ed., Springer Science and Business Media 519 pp.

Pitt, J.I., Hocking, A.D., Samson, R.A. and King, A.D. (1992) Recommended methods for mycological examination of foods. In : "Modern Methods in Food Mycology". Samson R. A., Hocking A. D., Pitt J. I. and King A. D. (Ed.), Elsevier, Amsterdam, 365-368.

Pons, W.A., Jr., Cucullu, A.F.,. Frenz, A.O, Jr., Lee, L.S. and Goldblatt, L.A. (1972) Rapid quantitative TLC method for determining aflatoxins in cottonseed products. Journal Association of Official Analytical Chemists, 55, 768-774.

Raper, K.B. and Fennell, D.I. (1965) "The Genus Aspergillus". Williams and Wilkins, Baltimore, Maryland, $686 \mathrm{pp}$.

Reddy, P.L. and Sreeramulu, A. (2016) Isolation and screening of amylolytic fungi from different soil samples of Chittoor district. J. Pharm. Biol. Sci., 4 (3), 92-95.

Riba, A., Bouras, N., Mokrane, S., Mathieu, F., Lebrihi, A. and Sabaou, N. (2010) Aspergillus section Flavi and aflatoxins in Algerian wheat and derived products. Food Chem. Toxicol , 48 (10), 2772-2777. 
Rustom, I.Y.S. (1997) Aflatoxin in food and feed: occurrence, legislation and inactivation by physical methods. Food Chemistry, 59 (1), 57-67.

Sekar, P., Yumnam, N. and Ponmurugan, K. (2008) Screening and characterization of mycotoxin producing fungi from dried fruits and grains. Adv. Biotech., 9 (7), 12-15.

Shivani, D. and Kumar, J.S. (2015) Extracellular enzymatic profile of fungal deteriogens ofHistorical Palace of Ujjain. Int. J. Curr. Microbiol. App. Sci. 4 (5), 122- 132.

Singh, S., Bali, V., Sharma, L. and Mangla, J. (2014) Production of Fungal Amylases Using Cheap, Readily Available Agriresidues, for Potential Application in Textile Industry. Bio Med Research International $9 \mathrm{pp}$.

Sweeney, M.J. and Dobson, A.D.W. (1998) Mycotoxin production by Aspergillus, Fusarium and Penicillium species. International Journal of Food Microbiology, $\mathbf{4 3}$ (3), 141-158.

Ulfig, J., Ulfig, K. and Markowska, A. (2009) Extracellular enzyme profiles of xerophilic fungi isolated from dried materials of medicinal plants. Pol. J. Environ. Stud. 18 (3), 391-397.

Wilson, J.P., Cooper, H.H. and Wilson, D.M. (1995) Effect of delayed harvest on contamination of pearl millet grain with mycotoxin producing fungi and mycotoxins. Mycopathology, 132 (1), 27-30.

Yin, Y., Lou, T., Jiang, J., Yan, L., Michailides, T. J. and Ma Z. (2008) Molecular characterization of toxigenic and a toxigenic Aspergillus flavus isolates collected from soil in various agroecosystems in China. J. Appl. Microbiol., 107 (6),1857-65. 
FUNGI ASSOCIATED WITH MAIZE AND SORGHUM GRAINS...

\title{
الفطريات المصاحبة لحبوب الذرة الثامية والذرة الرفيعة وقدرتها على أنتاج أنزيم الاميليز والافلاتوكسينات
}

\author{
محمد أحمد عبدالساتر 1، صبحي إبراهيم إسماعيل عبدالحافظ1 ، نعمات عبدالجواد

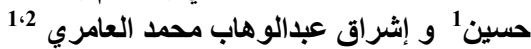

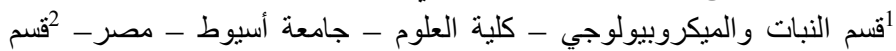 \\ الميكروبيولوجي - كلية العلوم التطبيقية - جامعة تعز - اليمن .
}

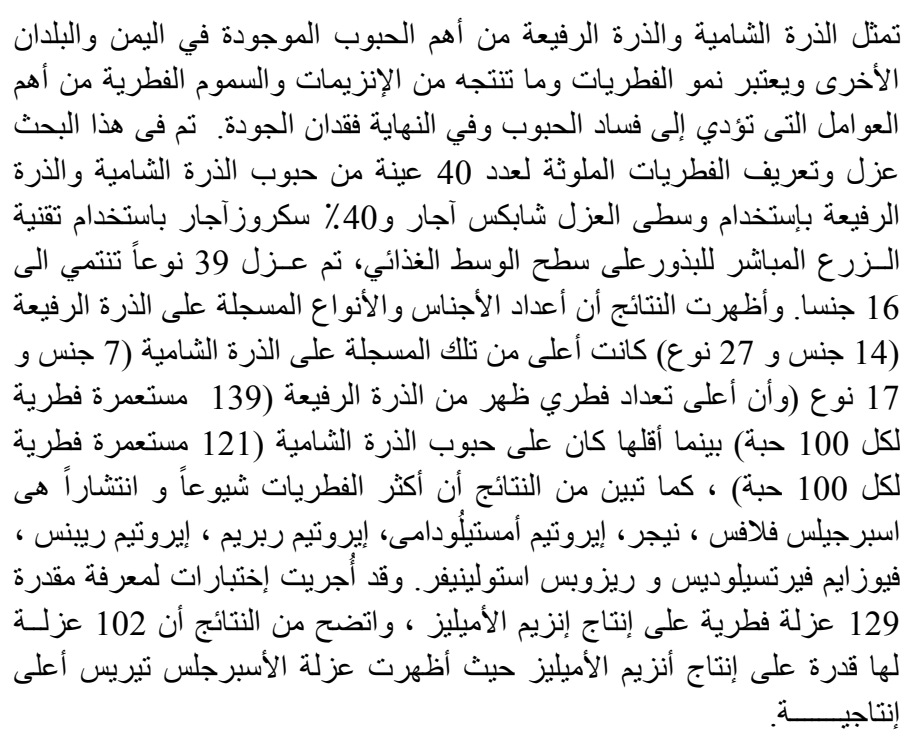

تم إجر اء تحليل HPLC لعدد 6 سلالات من فطرة الأسبرجلس فلافس لإختبار

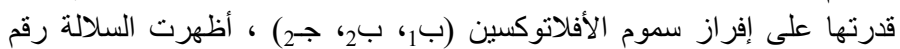
11311 AUMC 11317 AUMC

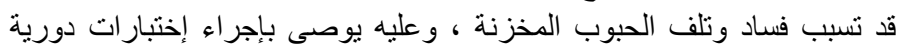
على الحبوب المخزنة لمعرفة الفطريات الملوثة وقدرنها ولفها على بلى إفراز الانزيمات

و السموم وذللك لتلافى فسادها. 